\title{
Numerical convergence of viscous-plastic sea ice models
}

\author{
Jean-François Lemieux ${ }^{1}$ and Bruno Tremblay ${ }^{1}$ \\ Received 11 July 2008; revised 31 December 2008; accepted 17 February 2009; published 12 May 2009.
}

[1] We investigate the convergence properties of the nonlinear solver used in viscousplastic (VP) sea ice models. More specifically, we study the nonlinear solver that is based on an implicit solution of the linearized system of equations and an outer loop (OL) iteration (or pseudo time steps). When the time step is comparable to the forcing time scale, a small number of OL iterations leads to errors in the simulated velocity field that are of the same order of magnitude as the mean drift. The slow convergence is an issue at all spatial resolution but is more severe as the grid is refined. The metrics used by the sea ice modeling community to assess convergence are misleading. Indeed, when performing $10 \mathrm{OL}$ iterations with a $6 \mathrm{~h}$ time step, the average kinetic energy of the pack is always within $2 \%$ of the fully converged value. However, the errors on the drift are of the same order of magnitude as the mean drift. Also, while 40 OL iterations provide a VP solution (with stress states inside or on the yield curve), large parts of the domain are characterized by errors of $0.5-1.0 \mathrm{~cm} \mathrm{~s}^{-1}$. The largest errors are localized in regions of large sea ice deformations where strong ice interactions are present. To resolve those deformations accurately, we find that more than $100 \mathrm{OL}$ iterations are required. To obtain a continuously differentiable momentum equation, we replace the formulation of the viscous coefficients with capping with a tangent hyperbolic function. This reduces the number of OL iterations required to reach a certain residual norm by a factor of $\sim 2$.

Citation: Lemieux, J.-F., and B. Tremblay (2009), Numerical convergence of viscous-plastic sea ice models, J. Geophys. Res., 114, C05009, doi:10.1029/2008JC005017.

\section{Introduction}

[2] Most sea ice dynamic models currently used in the community are based on a viscous-plastic (VP) formulation [Hibler, 1979]. The numerical schemes introduced over the years to solve the nonlinear sea ice momentum equation with a VP formulation can be broadly divided into two categories: the schemes that involve an implicit solution of the linearized system of equations and an outer loop (OL) iteration [e.g., Hibler, 1979; Tremblay and Mysak, 1997; Zhang and Hibler, 1997; Lemieux et al., 2008], hereafter referred to as VP models, and the ones that are based on a fully explicit time stepping scheme [e.g., Ip et al., 1991; Hunke and Dukowicz, 1997]. The original VP model [Hibler, 1979] is based on a modified Euler time step and a successive overrelaxation (SOR) solver. In this procedure, the nonlinear solution is first approximated by advancing the approximate solution to the middle of the time step by solving the linearized equation with an SOR solver. The nonlinear viscosities are then updated, the water drag is linearized using the newly calculated velocities, and the new linearized system of equations is again solved with the SOR solver. This process can be repeated (pseudo time steps) to improve the convergence of the approximate solution. Subsequent numerical improve-

\footnotetext{
${ }^{1}$ Department of Atmospheric and Oceanic Sciences, McGill University, Montreal, Quebec, Canada.

Copyright 2009 by the American Geophysical Union. 0148-0227/09/2008JC005017
}

ments to the original VP model mostly concentrated on the linear solver [e.g., Oberhuber, 1993; Zhang and Hibler, 1997; Lemieux et al., 2008]. Overall, these VP models share one common feature: their numerical scheme produces an approximate solution of the nonlinear momentum equation by solving implicitly (or partly implicitly) the linearized momentum equation a few times per time step. They might however differ in the terms treated explicitly or implicitly and in the method used to solve the linearized equation.

[3] While the convergence properties of linear solvers used in sea ice models are documented, those of the nonlinear solver have received relatively less attention. Some authors observed a slow response of VP models to changes in forcing. In the original VP model, Hibler [1979] showed that for a change in forcing, several (daily) time steps are required to attain equilibrium despite the fact that observations show a nearly instantaneous response (a few hours) to changes in forcing [Hunke and Zhang, 1999]. He recommended the use of a small time step compared to the scale of temporal variability of the forcing field. Ip [1993] and Zhang and Hibler [1997] have demonstrated that a single modified Euler time step does not lead to a VP solution; that is, a solution for which all the stress states lie either inside or on the yield curve. To get a VP solution, they propose to repeat the modified Euler time step many times per time step. Zhang and Hibler [1997] refer to pseudo time steps as the number of times the modified Euler time step is repeated within a time step.

[4] More recently, Hunke and Zhang [1999] have demonstrated the slow transient response of a VP model (which uses 
only the modified Euler time step and the previous time step solution as the initial guess) to changes in forcing. For example, in a case study of a low pressure system crossing the Arctic Ocean, they have shown that the VP model takes multiple (4 h) time steps to develop a cyclonic motion in the sea ice velocity field. The very slow response of the VP model is attributed to the fact that with a single modified Euler time step, the linearization causes the viscous coefficients to lag in time the forcing specified [see also Hunke and Dukowicz, 1997].

[5] As mentioned by Hunke [2001], many authors did not heed the advice about the convergence problem and used the model with a 1 day time step and daily varying winds [e.g., Hibler and Walsh, 1982; Riedlinger and Preller, 1991]. Some authors however recognized the problem and used many pseudo time steps to improve the level of convergence of the approximate solution [e.g., Geiger et al., 1998; Kreyscher et al., 2000].

[6] In a paper where the value of the sea ice compressive strength parameter $P^{*}$ was estimated from observations, Tremblay and Hakakian [2006] pointed out the two very different $P^{*}$ estimates derived in modeling studies by Hibler and Walsh [1982] $\left(P^{*}=27.5 \mathrm{kN} \mathrm{m}^{-2}\right)$ and Kreyscher et al. [2000] $\left(P^{*}=15 \mathrm{kN} \mathrm{m}^{-2}\right)$. In both studies, the optimal $P^{*}$ is obtained by minimizing the error between the buoy trajectories and the simulated drifts. Moreover, a larger $P^{*}$ in the work by Hibler and Walsh [1982] is required despite the fact that the magnitude of the surface wind stresses are nearly half as those used by Kreyscher et al. [2000]. Tremblay and Hakakian [2006] suggested that the discrepancy might in part be explained by the fact that the two models are not iterated to the same level of convergence: the model of Hibler and Walsh [1982] used a single modified Euler time step while Kreyscher et al. [2000] used 30 pseudo time steps (in both cases a 1 day time step is used).

[7] Up until now, the convergence of the approximate solution has been assessed by many authors using the average (or total) kinetic energy of the pack [e.g., Ip, 1993; Zhang and Hibler, 1997; Lemieux et al., 2008]. The states of stress have also often been used to evaluate the convergence of the approximate solution [e.g., Zhang and Hibler, 1997; Arbetter et al., 1999; Hunke and Zhang, 1999]. In this case, it is assumed that convergence is reached once all the states of stress lie either inside or on the yield curve. The specific goals of this paper are to revisit the numerical convergence of VP models, to determine the errors associated with various approximate solutions, to evaluate the metrics used by the sea ice modeling community to assess convergence and to investigate ways to improve the convergence properties and efficiency of VP sea ice models.

[8] The elastic-viscous-plastic (EVP) model developed by Hunke and Dukowicz [1997] is based on a fully explicit time stepping scheme. With this approach, there is an extra elastic term in the constitutive equation which allows for a larger sub-time step using a fully explicit time stepping scheme. This elastic term does not represent the physical elastic stress. It is an artificial term introduced in order to relax the stability condition with a fully explicit time stepping scheme. The study of the convergence properties of the EVP model is kept for future work.

[9] This paper is not intended to provide the acceptable magnitude of errors (which is probably application- dependent) for VP models but rather to document the convergence properties of VP models and suggest ways for improvements. It is structured as follows. In section 2, a review of the sea ice momentum equation with a VP formulation is given. In section 3 , we describe the nonlinear solver. In section 4 , we describe the model and the forcing fields used for the simulations. In section 5, we present results on the convergence properties of the nonlinear solver. In section 6, considerations are given to explain the slow convergence of the approximate solution. Concluding remarks and a description of future work are given in section 7 .

\section{Sea Ice Momentum Equation}

[10] The two-dimensional sea ice momentum equation is given by

$$
\rho_{i} h \frac{D \mathbf{u}}{D t}=-\rho_{i} h f \mathbf{k} \times \mathbf{u}+\boldsymbol{\tau}_{\boldsymbol{a}}-\boldsymbol{\tau}_{\boldsymbol{w}}+\nabla \cdot \boldsymbol{\sigma}-\rho_{i} h g \nabla H_{d},
$$

where $\rho_{i}$ is the density of the ice, $h$ the sea ice thickness, $f$ the Coriolis parameter, $\mathbf{u}$ the horizontal sea ice velocity vector, $\tau_{\boldsymbol{a}}$ the wind stress, $\boldsymbol{\tau}_{\boldsymbol{w}}$ the water drag, $\boldsymbol{\sigma}$ the internal ice stress tensor $(\nabla \cdot \sigma$ is defined as the rheology term), $g$ the gravity and $H_{d}$ the sea surface height. Following Tremblay and Mysak [1997], the sea surface tilt is expressed in terms of the geostrophic ocean current. Using a simple quadratic law with a constant turning angle, $\tau_{\boldsymbol{a}}$ and $\boldsymbol{\tau}_{\boldsymbol{w}}$ are expressed as [McPhee, 1975]

$$
\begin{gathered}
\boldsymbol{\tau}_{\mathbf{a}}=\rho_{a} C_{d a}\left|\mathbf{u}_{a}^{g}\right|\left(\mathbf{u}_{a}^{g} \cos \theta_{a}+\mathbf{k} \times \mathbf{u}_{a}^{g} \sin \theta_{a}\right), \\
\tau_{\mathbf{w}}=\rho_{w} C_{d w}\left|\mathbf{u}-\mathbf{u}_{w}^{g}\right|\left[\left(\mathbf{u}-\mathbf{u}_{w}^{g}\right) \cos \theta_{w}+\mathbf{k} \times\left(\mathbf{u}-\mathbf{u}_{w}^{g}\right) \sin \theta_{w}\right],
\end{gathered}
$$

where $\rho_{a}$ and $\rho_{w}$ are the air and water densities, $C_{d a}$ and $C_{d w}$ are the air and water drag coefficients, and $\mathbf{u}_{a}^{g}$ and $\mathbf{u}_{w}^{g}$ are the geostrophic wind and ocean current. Because $\mathbf{u}$ is much smaller than $\mathbf{u}_{a}$, $\mathbf{u}$ has been neglected in the expression for the wind stress.

[11] With a VP formulation, the constitutive law can be written as [Hibler, 1979]

$$
\sigma_{i j}=2 \eta \dot{\epsilon}_{i j}+[\zeta-\eta] \dot{\epsilon}_{k k} \delta_{i j}-P \delta_{i j} / 2, \quad i, j=1,2,
$$

where $\delta_{i j}$ is the Kronecker delta, $\dot{\epsilon}_{i j}$ are the strain rates defined by $\dot{\epsilon}_{11}=\frac{\partial u}{\partial x}, \dot{\epsilon}_{22}=\frac{\partial v}{\partial y}$ and $\dot{\epsilon}_{12}=\frac{1}{2}\left(\frac{\partial u}{\partial y}+\frac{\partial v}{\partial x}\right), \dot{\epsilon}_{k k}=\dot{\epsilon}_{11}+\dot{\epsilon}_{22}, \zeta$ is the bulk viscosity and $\eta$ is the shear viscosity. Following Hibler [1979], the pressure term $P$ (yield strength in isotropic compression) is parameterized by the following equation:

$$
P=P^{*} h \exp [-C(1-A)],
$$

where $P^{*}$ is the ice strength per meter, $A$ is the sea ice concentration and $C$ is the ice concentration parameter, an empirical constant characterizing the dependence of the compressive strength on sea ice concentration.

[12] The rheology term $(\nabla \cdot \sigma)$ depends on the yield curve and the flow rule, through the formulation of the bulk and 
shear viscosities. In the following, we use the elliptical yield curve with a normal flow rule [Hibler, 1979]. In this case, the bulk and shear viscosities are given by

$$
\begin{gathered}
\zeta=\frac{P}{2 \triangle}, \\
\eta=\zeta e^{-2},
\end{gathered}
$$

where $\Delta=\left[\left(\dot{\epsilon}_{11}^{2}+\dot{\epsilon}_{22}^{2}\right)\left(1+e^{-2}\right)+4 e^{-2} \dot{\epsilon}_{12}^{2}+2 \dot{\epsilon}_{11} \dot{\epsilon}_{22}(1-\right.$ $\left.\left.e^{-2}\right)\right]^{\frac{1}{2}}$, and $e$ is the ratio of the long axis and the short axis of the elliptical yield curve.

[13] In the limit where $\Delta$ tends to zero, equations (6) and (7) become singular. Following Hibler [1979], the values of $\zeta$ and $\eta$ are capped at maximum values of $\zeta_{\max }=\left(2.5 \times 10^{8}\right) P$ and $\eta_{\max }=\zeta_{\max } e^{-2}$. $\zeta$ and $\eta$ are also limited to minimum values of $\zeta_{\min }=4 \times 10^{8} \mathrm{~kg} \mathrm{~s}^{-1}$ and $\eta_{\min }=\zeta_{\min } e^{-2}$ in order to avoid potential numerical instabilities [Hibler, 1979]. Equations (6) and (7) therefore become

$$
\begin{gathered}
\zeta=\max \left(\min \left(\frac{P}{2 \triangle}, \zeta_{\max }\right), \zeta_{\min }\right), \\
\eta=\max \left(\min \left(\frac{P}{2 e^{2} \triangle}, \eta_{\max }\right), \eta_{\min }\right) .
\end{gathered}
$$

[14] A scale analysis of equation (1) for a time step of $6 \mathrm{~h}$ shows that the acceleration term is at least one order of magnitude smaller than typical wind stress values. For this reason, we neglect the acceleration term when a time step of $6 \mathrm{~h}$ is used. (Inertial and tidal oscillations are not included.) We have verified that solving the steady state momentum equation does not affect the conclusions presented in this paper. As we will see, the slow convergence is caused by the rheology term. Additional experiments are performed with a $30 \mathrm{~min}$ time step. In this case, the acceleration term is included (the advection of momentum term is however neglected) [Zhang and Hibler, 1997].

\section{Numerical Scheme}

[15] Because of the water drag term and especially the rheology term, equation (1) is strongly nonlinear. Indeed, sea ice resists virtually no load when the motion is divergent while it can resist large loads when convergence is present. At any given time step, the equation is linearized using an initial guess velocity field. The linear system of equations is then solved using the preconditioned Generalized Minimum RESidual (GMRES) method until a predefined convergence criterion is satisfied. The new solution is used to update the viscosities and $\left|\mathbf{u}-\mathbf{u}_{w}^{g}\right|$ in the water drag term. This process is repeated multiple times (OL) until a chosen convergence criterion is met for the nonlinear equation or until a fixed number of OL iterations have been performed. Note that there are two convergence criteria: one for the linear problem (that might change from one OL iteration to the next) and one for the nonlinear problem. In this paper, we refer to the linear convergence criterion as the tolerance.

[16] The momentum equation is discretized using finite differences. The $u$ and $v$ components of the velocity are positioned on the Arakawa $\mathrm{C}$ grid. A Dirichlet boundary condition is applied at an ocean-land boundary $(\mathbf{u}=0)$ and a Neumann condition at an open boundary (i.e., the spatial derivatives of the components of velocity in the normal direction with the open boundary are chosen to be zero). For stability, the pressure $P$ is set to zero at the open boundaries [Dukowicz, 1997]. Close to model boundaries, proper left and right difference schemes are used in the Taylor series expansion to evaluate the spatial derivatives. At the $k$ th OL iteration, the $u$ and $v$ momentum equations are written as

$$
\begin{aligned}
& \frac{\partial}{\partial x}\left[\left(\eta\left(\mathbf{u}_{l}^{k}\right)+\zeta\left(\mathbf{u}_{l}^{k}\right)\right) \frac{\partial u^{k}}{\partial x}\right]+\frac{\partial}{\partial y}\left[\eta\left(\mathbf{u}_{l}^{k}\right) \frac{\partial u^{k}}{\partial y}\right] \\
& \quad+\frac{\partial}{\partial x}\left[\left(\zeta\left(\mathbf{u}_{l}^{k}\right)-\eta\left(\mathbf{u}_{l}^{k}\right)\right) \frac{\partial v^{k}}{\partial y}\right]+\frac{\partial}{\partial y}\left[\eta\left(\mathbf{u}_{l}^{k}\right) \frac{\partial v^{k}}{\partial x}\right] \\
& \quad+\rho_{i} h f v_{a v g}^{k}-C_{w}\left(\mathbf{u}_{l}^{k}\right)\left(u^{k} \cos \theta_{w}-v_{a v g}^{k} \sin \theta_{w}\right)=b_{u}
\end{aligned}
$$

$$
\begin{aligned}
& \frac{\partial}{\partial y}\left[\left(\eta\left(\mathbf{u}_{l}^{k}\right)+\zeta\left(\mathbf{u}_{l}^{k}\right)\right) \frac{\partial v^{k}}{\partial y}\right]+\frac{\partial}{\partial x}\left[\eta\left(\mathbf{u}_{l}^{k}\right) \frac{\partial v^{k}}{\partial x}\right] \\
& \quad+\frac{\partial}{\partial y}\left[\left(\zeta\left(\mathbf{u}_{l}^{k}\right)-\eta\left(\mathbf{u}_{l}^{k}\right)\right) \frac{\partial u^{k}}{\partial x}\right]+\frac{\partial}{\partial x}\left[\eta\left(\mathbf{u}_{l}^{k}\right) \frac{\partial u^{k}}{\partial y}\right] \\
& \quad-\rho_{i} h f u_{a v g}^{k}-C_{w}\left(\mathbf{u}_{l}^{k}\right)\left(v^{k} \cos \theta_{w}+u_{a v g}^{k} \sin \theta_{w}\right)=b_{v} .
\end{aligned}
$$

where $b_{u}$ and $b_{v}$ are the sum of all the terms that do not depend on $\mathbf{u}^{k}, \mathbf{u}_{l}^{k}$ is the velocity vector used at the $k$ th iteration to linearize the momentum equation (the subscript $l$ refers to the linearization) and $v_{a v g}$ is the spatial averaging of the four neighboring $v$ components of velocity at the $u$ location (and vice versa for $u_{\text {avg }}$ ). An $\mathrm{f}$ plane approximation is used with $f=1.46 \times 10^{-4} s^{-1}$. In equations (10) and (11), $C_{w}\left(\mathbf{u}_{l}^{k}\right)$ is defined as

$$
C_{w}\left(\mathbf{u}_{l}^{k}\right)=\rho_{w} C_{d w}\left|\mathbf{u}_{l}^{k}-\mathbf{u}_{w}^{g}\right|
$$

[17] When using the previous iterate for linearization $\left(\mathbf{u}_{l}^{k}=\right.$ $\left.\mathbf{u}^{k-1}\right)$, Hibler and Ackley [1983] found a splitting problem under certain free drift conditions. They observed that the water drag terms were very different between the two steps of the modified Euler procedure. We follow Hibler and Ackley [1983], and express $\mathbf{u}_{l}^{k}$ with the past two iterates as

$$
\mathbf{u}_{l}^{k}=\frac{\left(\mathbf{u}^{k-1}+\mathbf{u}^{k-2}\right)}{2}, \quad k=2,3 ., k_{\max },
$$

where for $k=1, \mathbf{u}_{l}^{1}$ is the initial guess velocity $\left(\mathbf{u}^{0}\right)$.

[18] Because symmetry is not a prerequisite for the preconditioned GMRES method, all the terms in equations (10) and (11) are treated implicitly. The structure of the numerical scheme is as follows:

1. Start with an initial guess $\mathbf{u}^{0}$ do $k=1, k_{\text {ite }}$

2. Calculate $\zeta\left(\mathbf{u}_{l}^{k}\right), \eta\left(\mathbf{u}_{l}^{k}\right)$ and $C_{w}\left(\mathbf{u}_{l}^{k}\right)$

3. Calculate the initial residual norm $\left(R_{\text {ini }}^{k}\right)$ of the linearized system of equations 
4. Solve the linearized system of equations (equations (10) and (11)) for $u^{k}$ and $v^{k}$ using the preconditioned GMRES method. enddo

[19] We refer to this "do loop" as the OL. In step 4, the preconditioned GMRES method iterates until the tolerance is achieved, i.e., until the residual norm is lower than $R_{i n i}^{k} / \alpha(k)$. When different values of $\alpha(k)$ are used throughout the OL iteration process, we refer to this evolution of the tolerance as the progressive $\alpha(k)$ tolerance. The number of OL iterations performed is $k_{\text {ite. }}$. As mentioned, a convergence criterion for the nonlinear system of equations can also be used instead. Note that the OL iterations are similar to the pseudo time steps (two OL iterations correspond to one pseudo time step) introduced by Zhang and Hibler [1997] and to the OL iterations used by Tremblay and Mysak [1997]. The initial guess $\mathbf{u}^{0}$ can be the previous time step solution, a zero velocity field or the free drift solution. The preconditioned GMRES method is described by Lemieux et al. [2008]. The results presented here are independent of the linear solver used (provided the linear solvers are used with the same tolerance). For instance, one linear solver (e.g., the preconditioned GMRES method) which requires less cpu time to solve the linearized system of equations than another linear solver (e.g., the stand-alone SOR solver) will imply the same number of OL iterations to solve the nonlinear system of equations (up to a certain residual norm). Tests were performed with a stand-alone SOR solver to verify this.

\section{Model and the Forcing Fields}

[20] The model has a resolution of $10 \mathrm{~km}$. For all the simulations presented here, an elliptical yield curve and a normal flow rule are used. Conclusions drawn in this paper are believed to also apply to other yield curves and flow rules. For instance, the convergence properties of the model of Tremblay and Mysak [1997] exhibit similarities with results presented in this paper (results not shown). Note that Tremblay and Mysak [1997] used a Mohr-Coulomb yield curve with a nonnormal flow rule. Our model has two thickness categories and a zero-layer thermodynamic. More details on the thermodynamic can be found in the work by Tremblay and Mysak [1997]. The advection is performed at the end of the last OL iteration with a simple upstream scheme. A lower-resolution $(110 \mathrm{~km})$ version of the model is also sometimes used to perform additional tests.

[21] The wind stress is calculated from geostrophic winds derived from the National Center for Environmental Prediction and National Center for Atmospheric Research (NCEP/ NCAR) $6 \mathrm{~h}$ reanalysis of sea level pressure [Kalnay et al., 1996]. The climatological ocean currents were obtained from the steady state solution of the Navier-Stokes equation in which the advection of momentum is neglected, a 2-D nondivergent field is assumed and a quadratic drag law is used. The forcing used to derive the ocean currents is a 30 year climatological wind stress field. The sea ice model is coupled thermodynamically to a slab ocean model. The thermodynamics are forced by NCEP/NCAR reanalysis of monthly mean surface air temperature. All NCEP/NCAR reanalysis data are found at http://www.cdc.noaa.gov/.

[22] Starting with a constant sea ice thickness of $1 \mathrm{~m}$ and a concentration of $100 \%$, the model was run for 5 years from
1992 to 1996 . The fields obtained on 31 December 1996 are used as the initial conditions for the simulations presented here. The rheology parameters are taken as $P^{*}=30 \times 10^{3} \mathrm{~N}$ $\mathrm{m}^{-2}, C=20$ and $e=2$. Values for the other model parameters are the same as used by Tremblay and Mysak [1997].

\section{Simulation Results}

[23] Convergence properties of the numerical solver can be studied for particular time steps by performing a large number of OL iterations. They can also be studied by performing a fixed number of OL iterations per time step and looking at the evolution of the solution over many time steps. The previous time step solution is then used as the initial guess. Zhang and Rothrock [2000] have shown that for a fixed number of pseudo time steps, the approximate solution approaches a VP solution as the time step is reduced. This latter approach however involves a coupling between the continuity/energy equation and the dynamic through the thickness and concentration fields. We adopt here the former approach for most of the runs. This approach allows one, keeping the sea ice concentration and thickness fields constant throughout the iteration process, to focus on the convergence properties of the nonlinear solver in solving the sea ice momentum equation.

[24] We study the convergence properties for a fixed time step of $6 \mathrm{~h}$ (unless otherwise specified), 6 hourly varying wind stress and by performing a large number of $\mathrm{OL}$ iterations. The free drift (unless otherwise specified) solution is used as the initial guess. Lemieux et al. [2008] have shown that with a $6 \mathrm{~h}$ time step and 6 hourly varying wind stress, the free drift solution, as opposed to the previous time step solution, provides a better initial guess as it allows one to get, more efficiently, a better converged approximate solution. Some additional results with a time step of $30 \mathrm{~min}$ and using the previous time step solution as the initial guess are also given.

[25] Analysis were done for the month of January 1997. In the following, most of the results are for one particular time step (6 January 19970000 UT). The results for this time step are representative of the results obtained for other time steps. All real variables were defined as double precision. All simulations were performed on a desktop computer (2 quad Intel(R) Xeon(R) $2.33 \mathrm{GHz}$, cache of $4096 \mathrm{~Kb}$ with a RAM of $3.9 \mathrm{~Gb}$ ). The fortran compiler is gfortran 4.1.2, 64 bits. The optimization option $\mathrm{O} 3$-ffast -math was used for all the runs.

[26] Below, we refer to errors as the difference between an approximate solution of the velocity field and the fully converged (FC) velocity field solution. The FC solution is the best approximate solution as it is limited by the machine precision. It is however an overkilled approximate solution. A "sufficiently" converged approximate solution certainly does not require such an accuracy for typical applications of VP sea ice models. Unless otherwise specified, statistics for the errors are calculated and results are plotted for the part of the domain for which the sea ice concentration is higher than $50 \%$. Also, for clarity, only one out of a 100 velocity field vectors and one out of a 100 states of stress are shown on the plots (for $\zeta>\zeta_{\min }$ only). Note that for all the simulations performed, the tolerance of the linear solver (preconditioned GMRES method) was chosen to be low enough not to limit the convergence rate of the nonlinear approximate solution. 


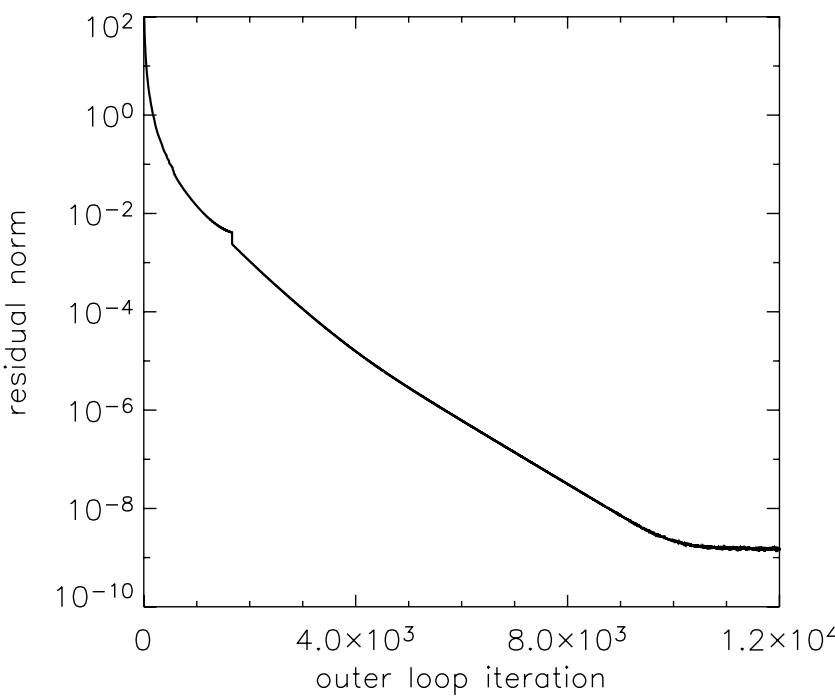

Figure 1. Residual norm of the nonlinear system of equations on 6 January 19970000 UT as a function of the outer loop (OL) iteration. The residual norm is calculated over the whole domain.

The optimal $\alpha(k)$ were found to be $\alpha(1)=1145, \alpha(2)=210$ and $\alpha(k)=200$ for $k$ greater than 2 . We refer to this tolerance evolution as the optimal progressive $\alpha(k)$ tolerance. Following Zhang and Hibler [1997], we refer to a VP solution as an approximate solution for which all the states of stress lie either inside or on the yield curve.

\subsection{Evolution of the Errors Throughout the OL Iteration Process}

[27] For the time step investigated (6 January 1997 $0000 \mathrm{UT}$ ), the number of OL iterations to obtain the FC solution is $\sim 10,500$ (see Figure 1). The residual norm after $\sim 10,500$ OL iterations is the smallest residual norm possible (which is limited by the machine precision). Tests performed with the $10-\mathrm{km}$ model show that typically $10,000 \mathrm{OL}$ iterations are required to reach the FC velocity field while 1000 OL iterations are needed with the $110-\mathrm{km}$ resolution model.

[28] The statistics of the errors as a function of the number of OL iterations are shown in Figures $2 \mathrm{a}$ and $2 \mathrm{~b}$. The domain average error (dashed curve on Figure 2a) drops relatively quickly because large parts of the domain, characterized by very small deformations (viscous phase), converge relatively fast. The maximum error (solid curve on Figure 2a) however decreases more slowly. The largest errors are located in narrower zones of large sea ice deformations. The fact that large parts of the domain have errors that converge relatively quickly as opposed to the errors in narrower regions (along large sea ice deformations as it is shown in section 5.4) is also observed on Figure $2 b$ that shows the distribution of the errors throughout the OL iteration process. Figure $2 \mathrm{~b}$ shows that $25 \mathrm{OL}$ iterations are required to have less than $1 \%$ of cells with errors larger than $1 \mathrm{~cm} \mathrm{~s}^{-1}$. As mentioned, this paper is not intended to provide the acceptable magnitude of errors. The criterion for the acceptable magnitude of the errors is probably application-dependent: for instance, the acceptable errors for a short-term forecast of sea ice conditions is likely to be different than for long-term climate simulations.

\subsection{Metrics to Assess Convergence of the Approximate Solution}

[29] We evaluate the common metrics to assess convergence of the nonlinear approximate solution. To evaluate the convergence of the approximate solution, we compare this solution to the FC solution. The FC velocity field and velocity errors for different number of OL iterations are shown on Figure 3 for 6 January 19970000 UT. Note that there are regions where the sea ice concentration is higher than $50 \%$ but for which the FC velocity vectors are very small and therefore are not visible (e.g., in the Canadian Arctic Archipelago). The FC average and maximum drifts on

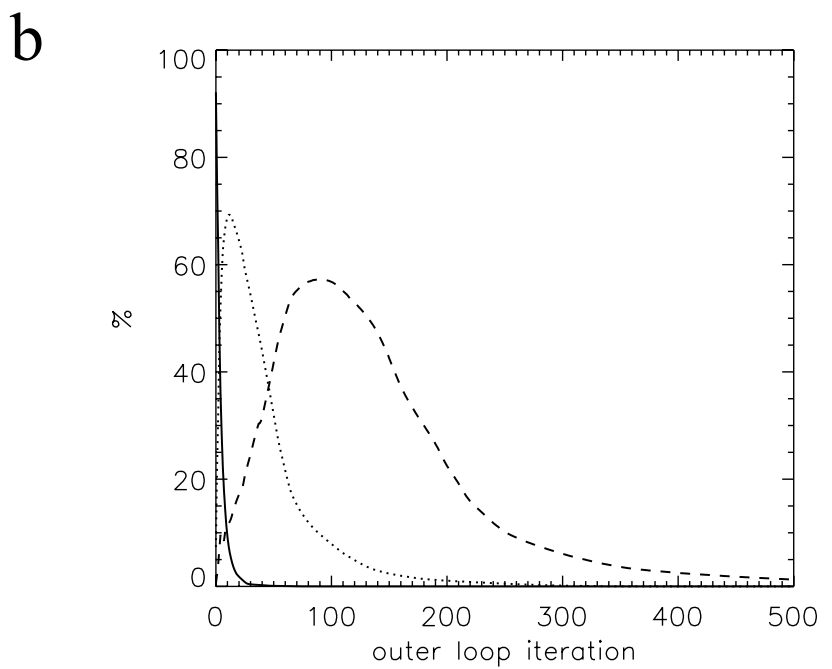

Figure 2. (a) Domain average (dashed curve) and maximum (solid curve) errors as a function of the number of OL iterations on 6 January 19970000 UT. (b) Distribution of the errors as a function of the number of OL iterations on 6 January 19970000 UT. Solid curve is error $\geq 1 \mathrm{~cm} \mathrm{~s}^{-1}$. Dotted curve is $1 \mathrm{~cm}$ $\mathrm{s}^{-1}>$ error $\geq 0.1 \mathrm{~cm} \mathrm{~s}^{-1}$. Dashed curve is $0.1 \mathrm{~cm} \mathrm{~s}^{-1}>$ error $\geq 0.01 \mathrm{~cm} \mathrm{~s}^{-1}$. 
a

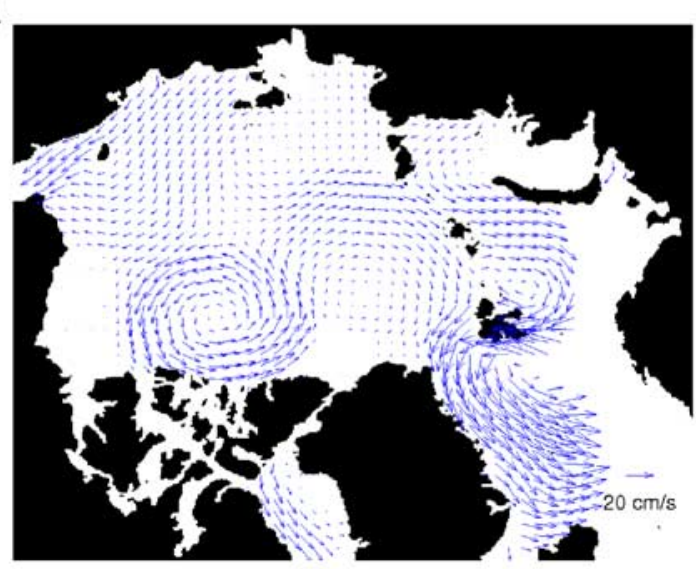

$\mathrm{C}$

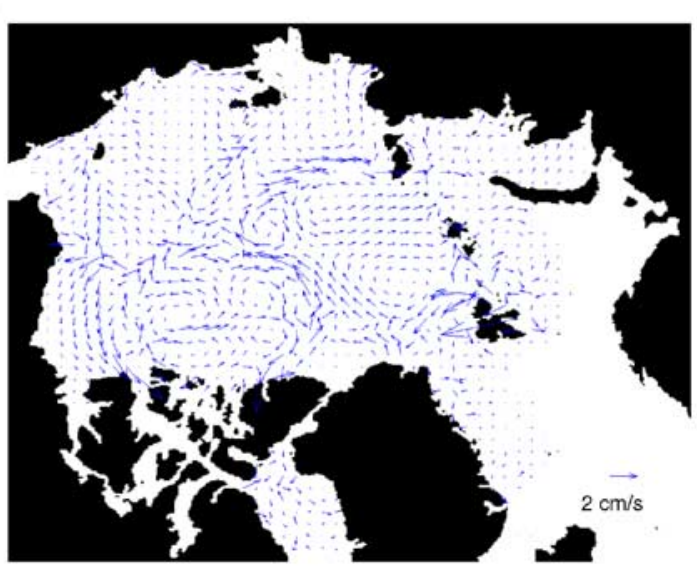

b

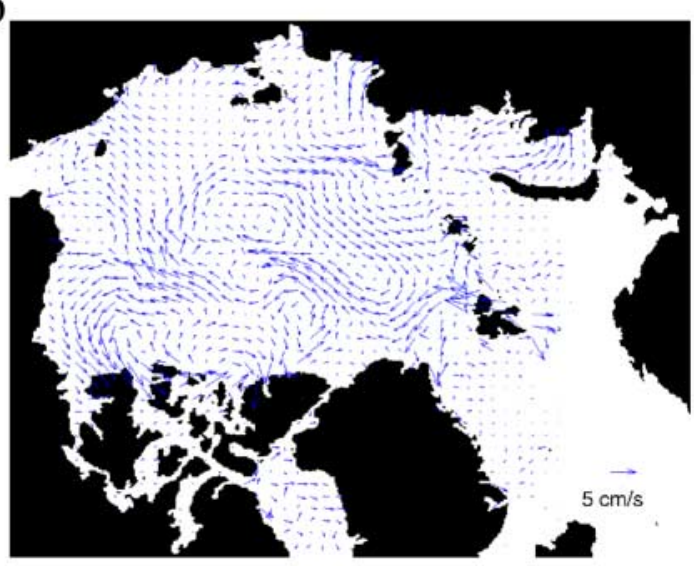

d

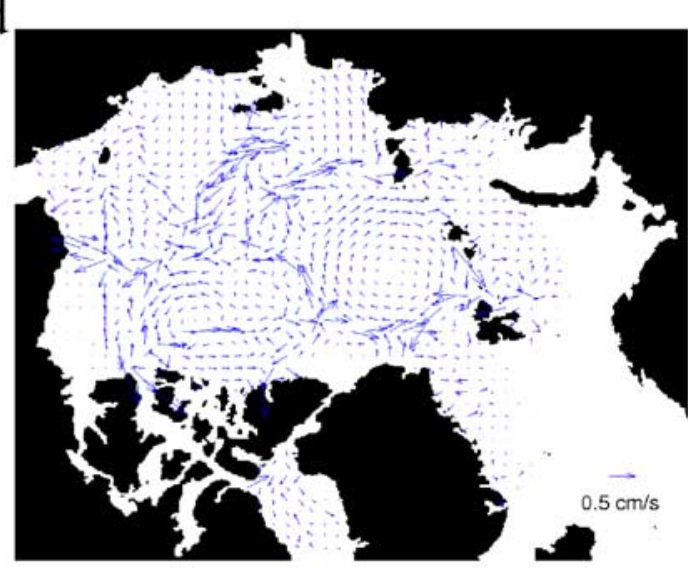

Figure 3. (a) Fully converged (FC) velocity field on 6 January 19970000 UT. Difference between the velocity field after (b) 2, (c) 10, and (d) $40 \mathrm{OL}$ iterations and the FC velocity field.

6 January 19970000 UT are $7.4 \mathrm{~cm} \mathrm{~s}^{-1}$ and $41.8 \mathrm{~cm} \mathrm{~s}^{-1}$, respectively.

[30] We recall that in the original VP model [Hibler, 1979], the numerical scheme is based on a modified Euler time step and an SOR solver. In this case, the linearized equation is solved twice per time step, which is equivalent to $2 \mathrm{OL}$ iterations with our approach. Figure $3 \mathrm{~b}$ shows the difference between the velocity field after $2 \mathrm{OL}$ iterations and the FC velocity field. Errors of the same order of magnitude as the mean drift are present in a large portion of the domain. Errors as large as $15 \mathrm{~cm} \mathrm{~s}^{-1}$ are found close to Svalbard and errors of $1-5 \mathrm{~cm} \mathrm{~s}^{-1}$ are present in large regions of the central Arctic. The lack of convergence of the approximate solution is also present in the normalized states of stress shown in Figure $4 \mathrm{~b}$. Many states of stress are neither viscous nor plastic, and are unrealistic as they lie outside of the yield curve. Note that these results remain the same when this experiment is repeated with a stand-alone SOR solver (as used by Hibler [1979]).

[31] Some authors have used the average (or total) kinetic energy (KE) of the ice pack to assess the convergence of the approximate solution [e.g., Ip, 1993; Zhang and Hibler, 1997; Lemieux et al., 2008]. Lemieux et al. [2008] found that when performing $10 \mathrm{OL}$ iterations, the average KE of the approximate solutions (for all the January 1997 time steps) is always within $2 \%$ of the $\mathrm{FC}$ average KE. The average KE is given by $\frac{1}{N} \sum_{j=1}^{N} \frac{\rho_{i} h_{j}\left(u_{j}^{2}+v_{j}^{2}\right)}{2}$, where $\mathrm{N}$ is the number of icecovered grid cells (concentration larger than 50\%) and $u$ and $v$ are the components of velocity interpolated at the tracer point. For the time step investigated here (6 January 1997 0000 UT), Figure 5 shows the average KE, normalized by the $\mathrm{FC}$ value, as a function of the number of OL iterations. Figure 5 indicates that in only three OL iterations, the average $\mathrm{KE}$ is within $2 \%$ of the $\mathrm{FC}$ value. Note that for this specific time step, the average $\mathrm{KE}$ after $10 \mathrm{OL}$ iterations is within $0.2 \%$ of the FC value. From Figure 5, one could conclude that the nonlinear approximate solution converges very efficiently. This is however not the case. Figure $3 \mathrm{c}$ shows the difference between the velocity field obtained after $10 \mathrm{OL}$ iterations and the $\mathrm{FC}$ velocity field. Even though the average $\mathrm{KE}$ is within $0.2 \%$ of the FC value, Figure $3 \mathrm{c}$ indicates that large errors, of the same order of magnitude as the mean drift, are still present in the velocity field. Errors as large as $6 \mathrm{~cm} \mathrm{~s}^{-1}$ are found close to Svalbard and errors of $1-2 \mathrm{~cm} \mathrm{~s}^{-1}$ are present in large regions of the central Arctic. Again, the states of stress confirm that the approximate solution is not a perfect VP solution (Figure 4c).

[32] The position of the states of stress in principal stress space (or stress-invariant space) is a metric often used by modelers [e.g., Ip, 1993; Zhang and Hibler, 1997; Hunke and Dukowicz, 2002] to confirm that the approximate solution 
a

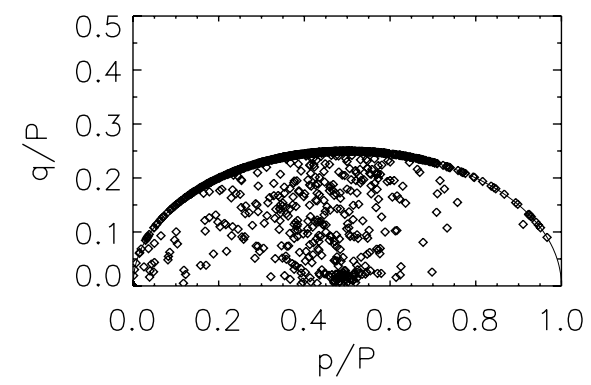

C

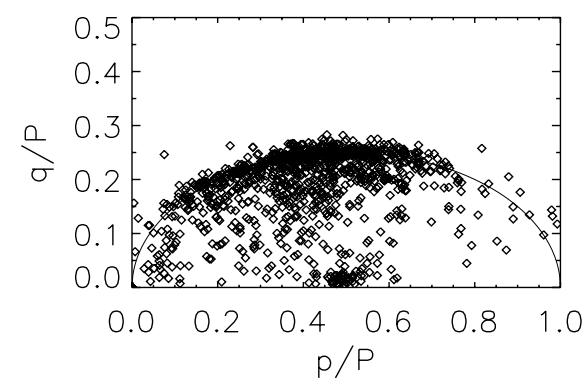

b

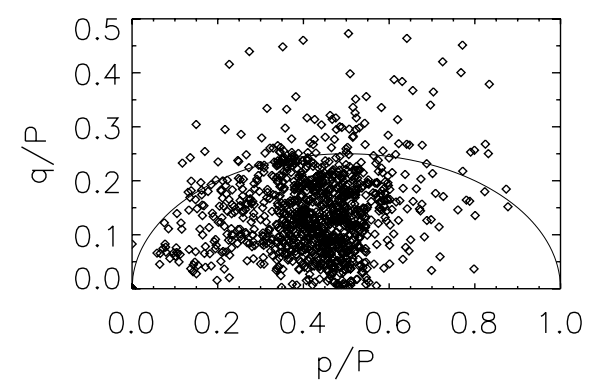

d

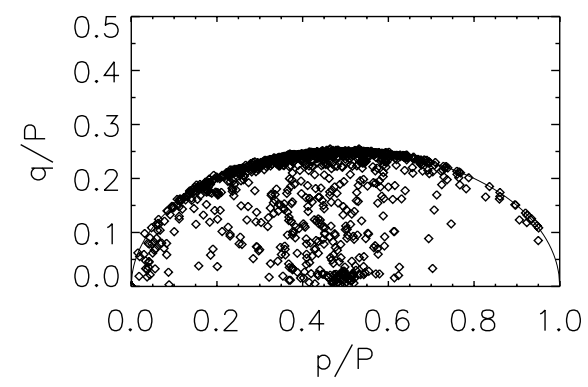

Figure 4. States of stress in stress-invariant space after (a) 10,500, (b) 2, (c) 10, and (d) 40 OL iterations on 6 January 19970000 UT. The stresses are normalized by $P$. The stress invariants are $p$ (the negative average of the normal stresses) and $q$ (the maximum shear stress).

has converged. We consider the approximate solution to be a VP solution if $99 \%$ of the states of stress are characterized by $0 \leq p \leq 1$ (first stress-invariant, normalized by $P$ ) and $0 \leq q \leq f(p)+\epsilon$ (second stress-invariant, normalized by $P$ ) where $f(p)$ is the analytical equation defining the ellipse. The parameter $\epsilon$ is chosen to be 0.005 . We find that $40 \mathrm{OL}$ iterations are needed to get a VP solution (Figure 4d). This can be compared to Figure 4a which shows the states of stress of the FC velocity field. After $40 \mathrm{OL}$ iterations, there are errors larger than $1 \mathrm{~cm} \mathrm{~s}^{-1}$ at some locations and large parts of the domain are characterized by errors of $\sim 0.5 \mathrm{~cm}$ $\mathrm{s}^{-1}$ (Figure 3d).

[33] The quality of the approximate solution however not only depends on the number of OL iterations but also on the time step compared to the forcing time scale (when using the previous time step solution as the initial guess). Zhang and Rothrock [2000] have shown that with a single modified Euler time step, the approximate solution approaches a VP solution as the time step is reduced. In some applications (e.g., when a VP model is coupled to an ocean model), the time step used is much smaller than $6 \mathrm{~h}$. For example, a time step of $30 \mathrm{~min}$ is used in the Goddard Institute for Space Studies General Circulation Model [Schmidt et al., 2006]. This is done because the sea ice component is less computationally intensive than the ocean/atmosphere components, and it is typically run at the ocean time step. When using such a small time step, the changes in the forcing field (from the atmosphere and ocean) are smaller and the previous time step solution is a much better initial guess.

[34] To address this, we have performed an additional test with a time step of $30 \mathrm{~min}$ and using the previous time step solution as the initial guess. In this case, the geostrophic wind field to force the model is linearly interpolated between two $6 \mathrm{~h}$ geostrophic wind fields. In this test, the sea ice advection and thermodynamic are turned off in order to focus on the convergence properties of the nonlinear solver. With a $30 \mathrm{~min}$ time step, neglecting the acceleration is no longer valid. The acceleration term is therefore included. Figure 6a shows the difference between the velocity field after $2 \mathrm{OL}$ iterations and the FC velocity field (on 6 January 19970000 UT). This can be compared to Figure $3 \mathrm{~b}$ (notice the different reference vector). The smaller time step leads to smaller errors over

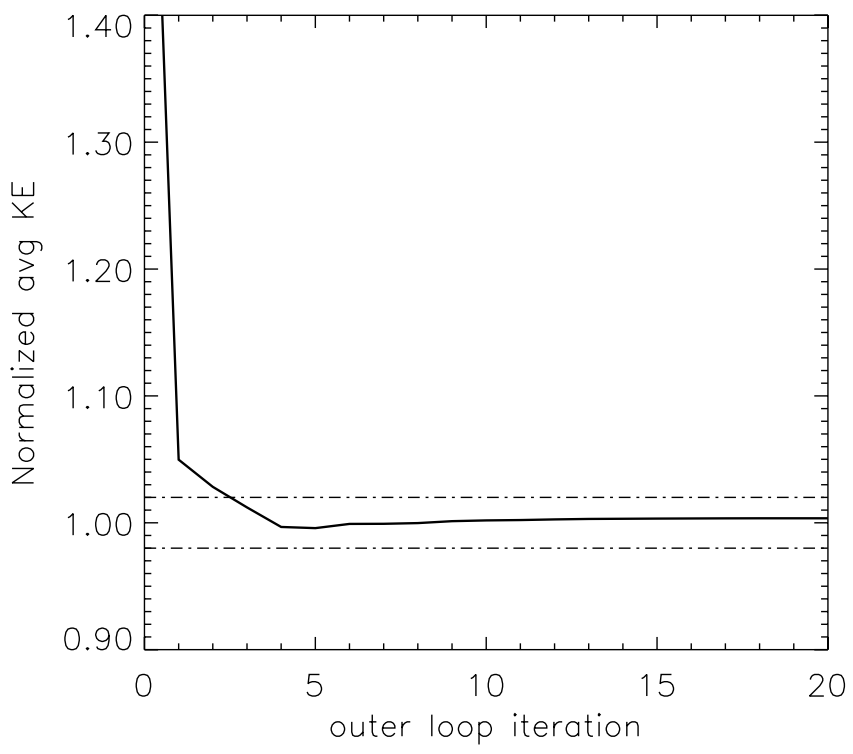

Figure 5. Average kinetic energy (KE) of the ice pack normalized by the FC KE of the ice pack as a function of the number of OL iterations for 6 January 19970000 UT. The two dashed-dotted lines indicate the $2 \%$ criterion. 
a

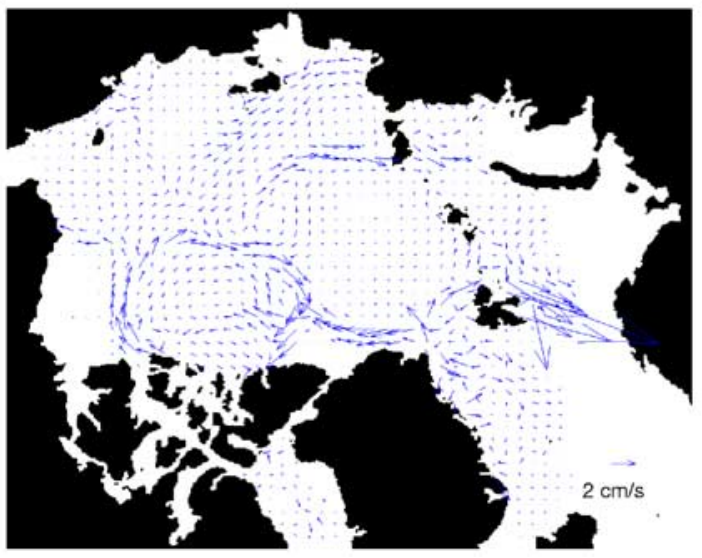

b

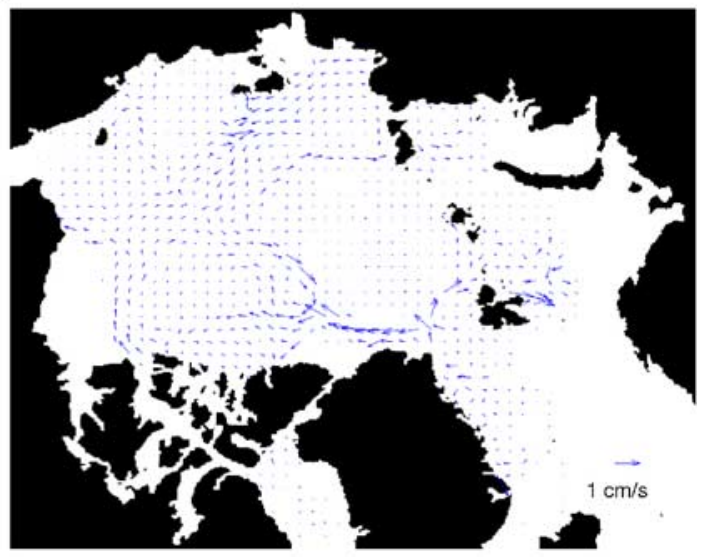

Figure 6. Difference between the velocity field after (a) 2 and (b) 10 OL iterations and the FC velocity field on 6 January 19970000 UT when using a 30 min time step.

most of the domain (the domain average error is $0.41 \mathrm{~cm} \mathrm{~s}^{-1}$ instead of $1.62 \mathrm{~cm} \mathrm{~s}^{-1}$ with the $6 \mathrm{~h}$ time step). There are however errors of $1-2 \mathrm{~cm} \mathrm{~s}^{-1}$ in many regions and larger errors close to Svalbard. Figure 6b shows the difference between the velocity field after $10 \mathrm{OL}$ iterations and the FC velocity field (on 6 January 19970000 UT). This can be compared to Figure $3 \mathrm{c}$ (notice the different reference vector). Again, the errors are smaller than with the $6 \mathrm{~h}$ time step case over most of the domain (the domain average error is $0.11 \mathrm{~cm}$ $\mathrm{s}^{-1}$ instead of $0.45 \mathrm{~cm} \mathrm{~s}^{-1}$ with the $6 \mathrm{~h}$ time step). As in the work by Zhang and Rothrock [2000], we have verified that with two OL iterations, the approximate solution approaches a VP solution as the time step is reduced (results not shown).

\subsection{Are the Errors Random or Systematic?}

[35] In section 5.2, it is shown that with a time step of $6 \mathrm{~h}$, large residual errors are present in the velocity field approximate solution when a small number of OL iterations are performed. In this section, we evaluate the impact of these errors on the mean sea ice drift. In particular, we want to know if these errors are systematic or random. Systematic errors are important because they create biases in the simulated fields (e.g., ice export, divergence). Because the momentum and continuity/energy equations are coupled (through the thickness and concentration fields), random errors can still significantly affect long-term simulations. Ultimately however, the test on the convergence should be done in a global climate model or other models depending on the application.

[36] In a first experiment, two simulations for the month of January 1997 were performed: one with two OL iterations and one with $1000 \mathrm{OL}$. We refer to the latter simulation as FC even though we know from Figure 1 that 1000 OL iterations are not sufficient to get a solution limited by the machine precision. We do this because of constraints on the total integration time. For these two simulations, the sea ice thermodynamic and the advection were not performed. The thickness and concentration fields are therefore the same throughout the whole month of integration. When comparing the monthly mean velocity fields (results not shown), the differences are smaller than for an individual snapshot (see Figure $3 \mathrm{~b}$ ) but they are still $\sim 1 \mathrm{~cm} \mathrm{~s}^{-1}$ over large regions of the Arctic, with the largest errors equal to $6.7 \mathrm{~cm} \mathrm{~s}^{-1}$. This indicates that systematic errors are present in the monthly mean velocity field (with two OL iterations, 6 hourly varying wind stress and a $6 \mathrm{~h}$ time step).

[37] In a second experiment, two simulations for the month of January 1997 were again performed: one with two OL iterations and one with 1000 OL. However, in this case, the dynamic is coupled to the thermodynamic and the advection is performed at the end of the last OL iteration for each time step. This allows one to assess the impact of the errors in simulating monthly mean fields.

[38] Figure 7a shows the difference between the January 1997 mean velocity field obtained with two and 1000 OL iterations (second experiment). The average drift of the FC solution is $5.5 \mathrm{~cm} \mathrm{~s}^{-1}$. As in the first experiment, large regions of the Arctic are characterized by errors of $\sim 1 \mathrm{~cm}$ $\mathrm{s}^{-1}$. The largest errors is $4.4 \mathrm{~cm} \mathrm{~s}^{-1}$. Simulations performed with the $110-\mathrm{km}$ resolution model show that these results are robust and do not depend on the particular month. Figure 7a also indicates a faster transpolar drift stream, a larger flux through Fram strait and more convergence north of the Canadian Arctic Archipelago (CAA) when the velocity field is not iterated to convergence. Figure $7 \mathrm{~b}$ shows the difference between the January 1997 mean thickness field when two and 1000 OL iterations are performed. Differences between the two fields can be as high as a few meters in localized regions (a few grid cells) but are capped to $\pm 0.5 \mathrm{~m}$ for clarity. There are significant differences in thickness in many regions of the domain. For instance, the use of two OL iterations results in more sea ice convergence north of the CAA and north of Greenland. This creates larger thickness buildup (in yellow, orange, and red). The anomalous advection of sea ice near the coast causes negative thickness differences (in blue) just north of these regions.

[39] When using a $30 \mathrm{~min}$ time step (and the previous time step solution as the initial guess), two OL iterations lead to monthly mean fields closer to the FC solutions. We refer here to the FC solution as the solution obtained with $60 \mathrm{OL}$ iterations. Only $60 \mathrm{OL}$ iterations are performed because of constraints on the total integration time. Figure $7 \mathrm{c}$ shows the difference between the January 1997 mean velocity field obtained with two and 60 OL iterations when using a $30 \mathrm{~min}$ 
a

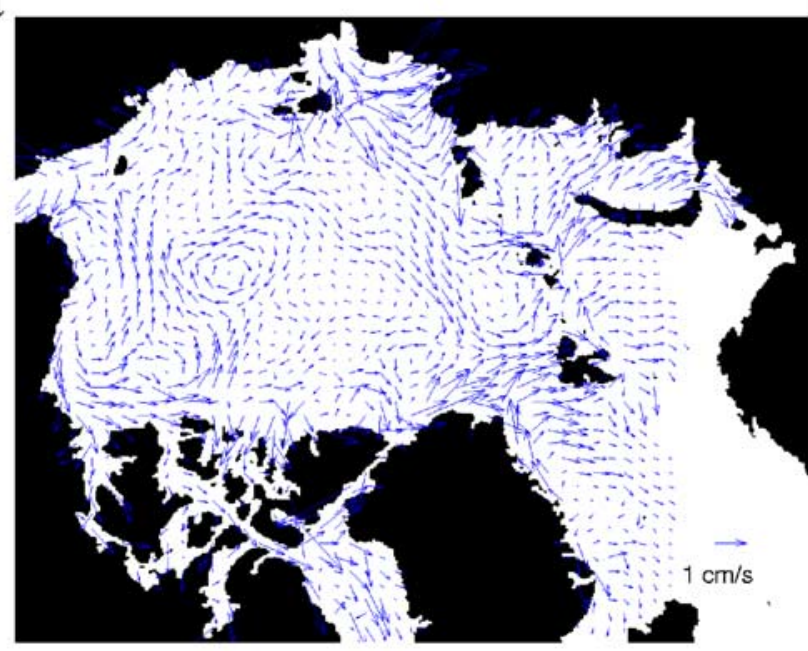

b

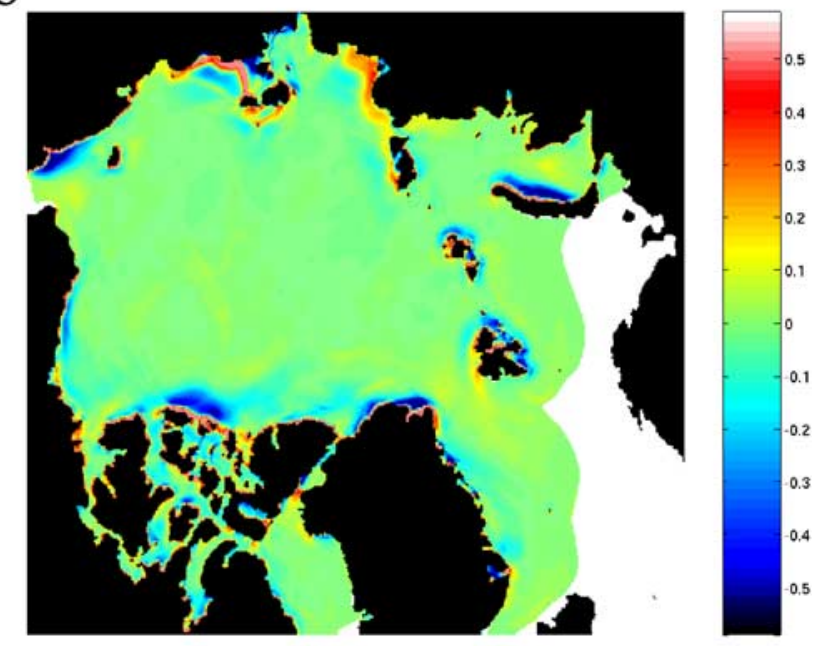

d

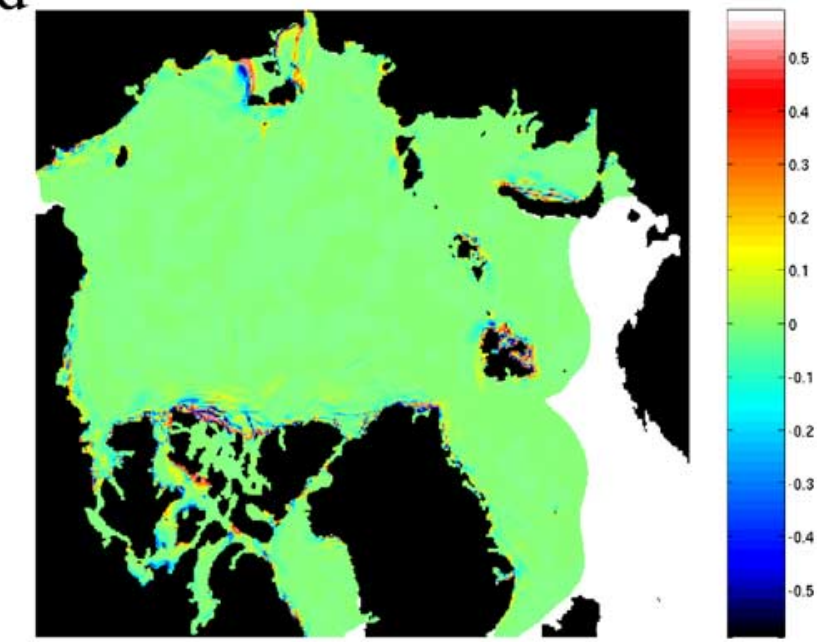

C

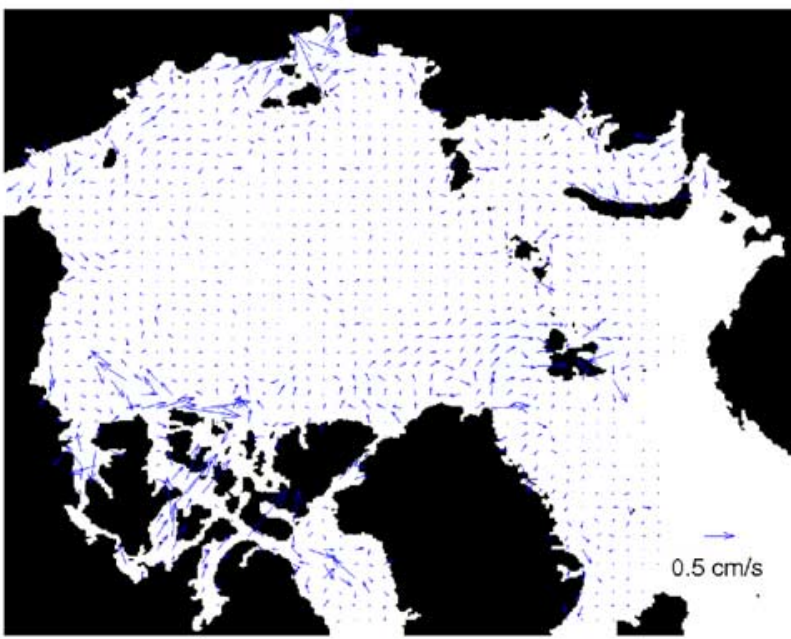

Figure 7. (a) Difference between the mean January 1997 velocity field obtained with 2 and 1000 OL iterations when using a $6 \mathrm{~h}$ time step. (b) Difference between the mean January 1997 thickness field obtained with 2 and 1000 OL iterations when using a $6 \mathrm{~h}$ time step. (c) Difference between the mean January 1997 velocity field obtained with 2 and 60 OL iterations when using a 30 min time step. (d) Difference between the mean January 1997 thickness field obtained with 2 and 60 OL iterations when using a 30 min time step.

time step. This can be compared with Figure 7 a (note however the different reference vector). The smaller time step leads to smaller errors over most of the domain (the domain average error is $0.1 \mathrm{~cm} \mathrm{~s}^{-1}$ instead of $0.5 \mathrm{~cm} \mathrm{~s}^{-1}$ with the $6 \mathrm{~h}$ time step and the maximum error is $2.8 \mathrm{~cm} \mathrm{~s}^{-1}$ instead of $4.4 \mathrm{~cm} \mathrm{~s}^{-1}$ with the $6 \mathrm{~h}$ time step). Figure $7 \mathrm{~d}$ shows the difference between the January 1997 mean thickness field when two and 60 OL iterations are performed. As in the $6 \mathrm{~h}$ time step case, differences between the two fields can be as high as a few meters in localized regions (a few grid cells) but are capped to $\pm 0.5 \mathrm{~m}$ for clarity. The thickness anomaly field has similarities with the one obtained with a $6 \mathrm{~h}$ time step (with large anomalies for example north of the CAA). However, the high thickness anomaly regions are more localized than in the $6 \mathrm{~h}$ time step case. Note that the atmospheric temperatures are prescribed in this model. The effect of the errors for a fully coupled model remains to be investigated.

\subsection{Evolution of Sea Ice Deformations Throughout} the OL Iteration Process

[40] Figures $3 b-3 d$ suggest some structure in the error field. To gain insight into the evolution of the errors, we compare the shear deformation field at different stages of the iteration process with the FC shear deformation field. Figure 8a shows the FC shear deformation field. The shear deformation (second strain rate-invariant) is defined as $\gamma=\sqrt{\left(\frac{\partial u}{\partial x}-\frac{\partial v}{\partial y}\right)^{2}+\left(\frac{\partial u}{\partial y}+\frac{\partial v}{\partial x}\right)^{2}}$. As seen in the work by Maslowski and Lipscomb [2003] for a model with about the same spatial resolution $(9 \mathrm{~km})$, the model simulates basinscale linear kinematic features (LKFs) that resemble the observed LKFs [Kwok, 2001].

[41] The importance to properly simulate the deformations of the sea ice cover is more and more recognized [e.g., Hutchings et al., 2005; Kwok et al., 2008]. Shear lines, 
a

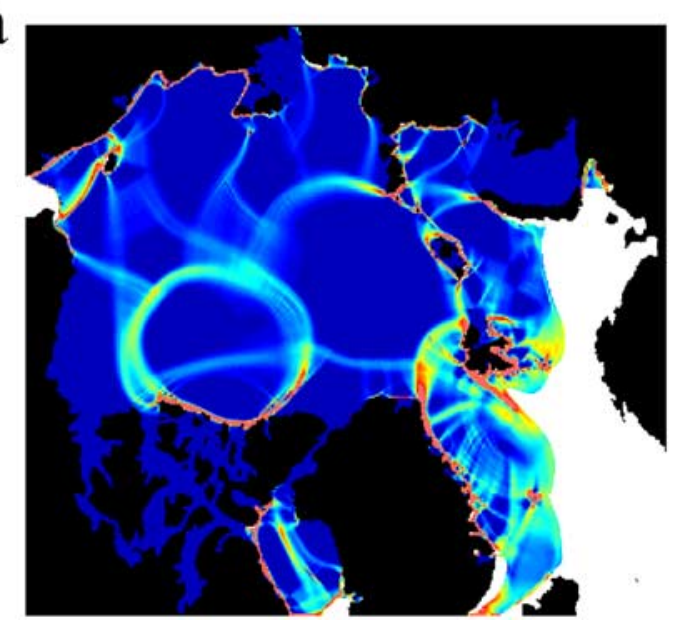

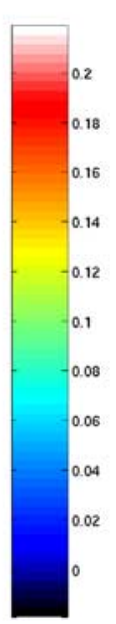

$\mathrm{b}$

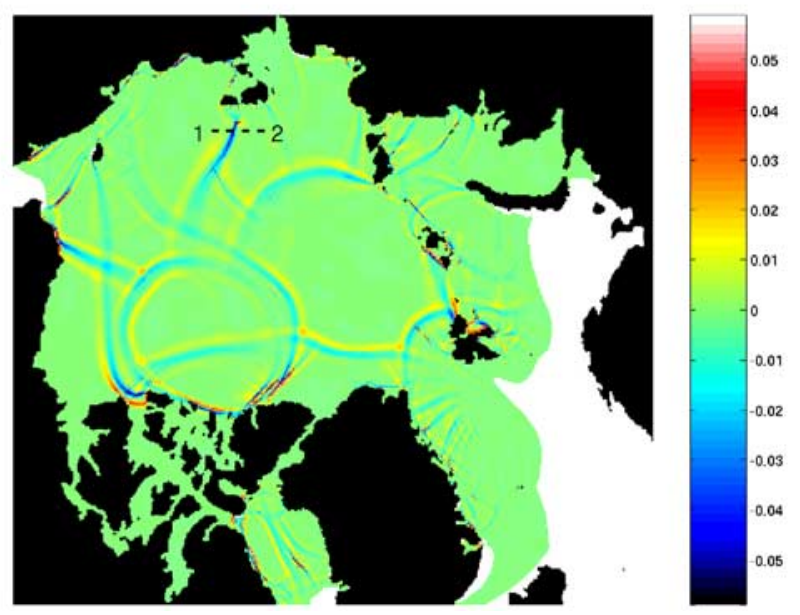

Figure 8. (a) Shear deformation (capped at 0.2 day $^{-1}$ for clarity) field on 6 January 19970000 UT for the FC solution. (b) Difference (capped at \pm 0.05 day $^{-1}$ for clarity) between the shear deformation field obtained after 40 OL iterations and the FC shear deformation field on 6 January 19970000 UT. The dashed black line between the points 1 and 2 defines a transect along which the shear deformation is plotted in Figure $9 \mathrm{~b}$.

pressure ridges and leads (LKFs) are important physical processes affecting sea ice thickness, air-sea heat, and moisture fluxes and ocean salt flux. Divergence/convergence of the sea ice cover drives changes in the thickness. For instance, pressure ridges are formed when convergence is present in regions of high sea ice concentration. Leads are created when divergence is present in the sea ice cover, allowing new ice to form (in Winter) or heat to be absorbed by the ocean surface layer (in Summer). McPhee et al. [2005] have observed an intense zone of pycnocline upwelling and measured large upward turbulent heat flux in the ocean boundary layer in a region of important sea ice shear deformations. McPhee et al. [2005] attributed this upwelling to Ekman pumping associated to the localized high shear strain rates in the sea ice velocity field. Overall, sea ice deformations largely affect the exchange of heat, moisture and momentum between the ocean and the atmosphere in the polar regions. Because deformations are calculated from spatial gradients of the velocity field, small errors in the velocities can have a large effect on the simulated deformations.

[42] After $10 \mathrm{OL}$ iterations, the shear deformation field roughly resembles the FC one but does not have all the fine structure and the right spatial localization of the shear lines (results not shown). After $40 \mathrm{OL}$ iterations, the shear deformation field is better defined and large rigid plates (characterized by low deformations) with zones of high deformations between them are more and more discernable (results not shown). Figure $8 \mathrm{~b}$ shows the difference between the shear deformation field after $40 \mathrm{OL}$ iterations and the FC shear deformation field on 6 January 19970000 UT. Figure $8 \mathrm{~b}$ shows regions of negative and positive anomalies of shear adjacent to the existing FC shear lines. This means that the location of the shear lines after $40 \mathrm{OL}$ iterations are spatially off compared to the FC shear lines and not as well defined. During the rest of the iteration process, more refinement and a better spatial localization of the deformations develop. This is also observed in the divergence field (results not shown).
[43] To illustrate the changes in the shear deformation field throughout the iteration process, we have calculated the distribution of shear in a $2000 \mathrm{~km}$ by $2000 \mathrm{~km}$ square region in the central Arctic (where multiyear ice is usually present). The distributions on Figure 9a consist of 150 bins of the shear deformation with intervals of $10^{-3} \mathrm{day}^{-1}$. Figure 9a shows the percentage of cells within each interval after 10 (blue curve, KE converged), 40 (red curve, VP solution) and 10,500 OL iterations (black curve, the FC solution). These three curves show that the distribution changes during the OL iteration process with a shift from medium size deformations $\left(0.01\right.$ day $\left.^{-1}<\gamma<0.07 \mathrm{day}^{-1}\right)$ to smaller deformations $(\gamma<$ 0.01 day $\left.^{-1}\right)$ and larger deformations $\left(\gamma>0.07\right.$ day $\left.^{-1}\right)$.

[44] We further analyze this issue of refinement of shear lines with increasing number of OL iterations by plotting the shear deformation along a transect crossing a shear line that develops north of Novosibirsk island (see dashed black line between points 1 and 2 on Figure $8 b$ ). Figure $9 \mathrm{~b}$ shows the value of shear deformation along this $400 \mathrm{~km}$ long transect after 10 (blue curve, KE converged), after 40 (red curve, VP solution), after 100 (green curve), and after 10,500 OL iterations (black curve, the FC solution). Figure $9 \mathrm{~b}$ shows that with 10, 40 and $100 \mathrm{OL}$ iterations, the definition of the shear line and the maximum value of $\gamma$ are still significantly different than the FC solution. Depending on the case (other transects), between 100 and $500 \mathrm{OL}$ iterations are needed for the maximum shear along the transect to reach $95 \%$ of the FC maximum shear. From Figures $8 \mathrm{~b}$ and $9 \mathrm{~b}$, we observe that as the number of OL iterations increases, the simulated sea ice cover is more and more characterized by large rigid plates with localized zones of high deformations between them.

[45] The impact of "poorly" simulated deformations (which would lead to errors in the simulated heat/moisture/ salt fluxes) on climate simulations remains to be investigated. In a recent paper, Kwok et al. [2008] found that current sea ice models systematically underestimate deformations (particularly divergence) when compared to Radarsat derived deformations. We raise the question whether the discrepancy between data and simulated deformations could be partly 
a

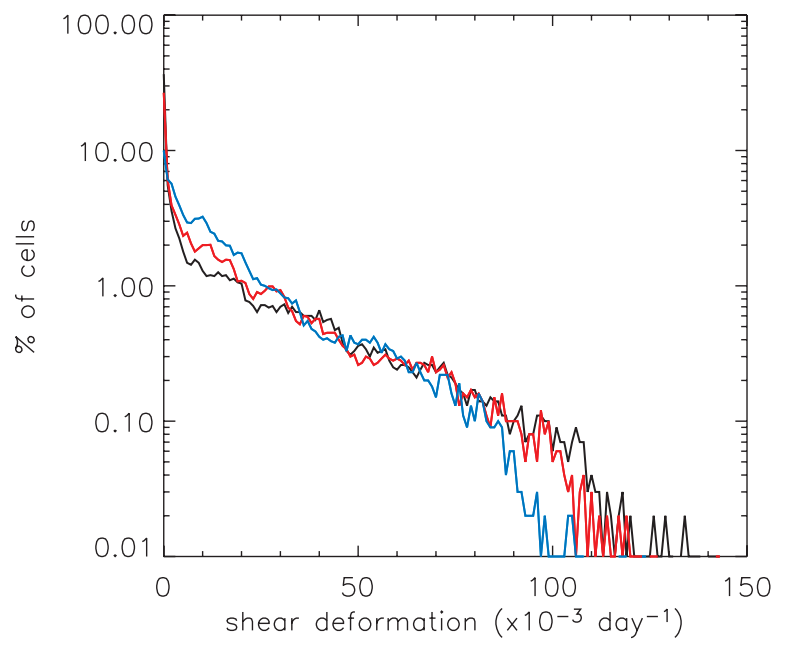

b

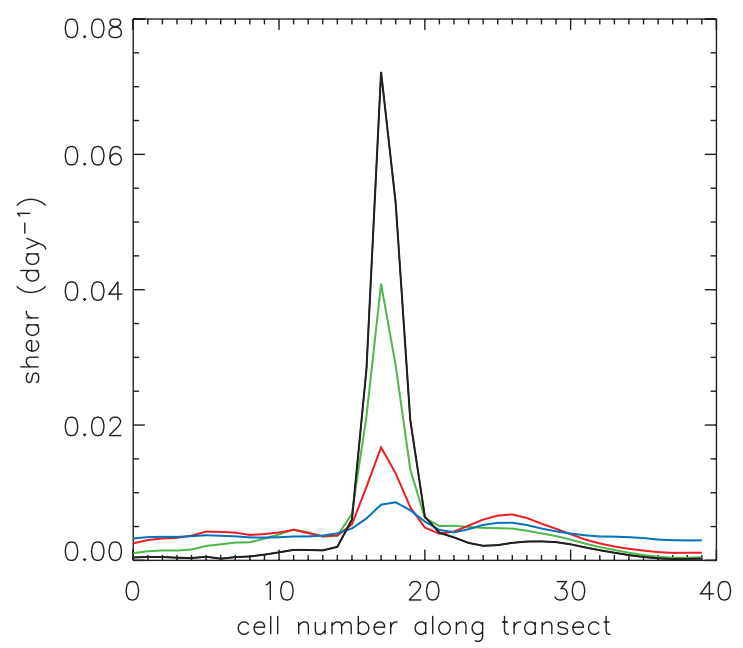

Figure 9. (a) Distribution of shear deformation on 6 January 19970000 UT after 10 (blue curve), 40 (red curve), and 10,500 (black curve) OL iterations. The bin interval is $10^{-3} \mathrm{day}^{-1}$. The distributions are calculated for a $2000 \mathrm{~km} \times 2000 \mathrm{~km}$ square region in the central Arctic. (b) Shear deformation along the $400 \mathrm{~km}$ transect after 10 (blue curve), 40 (red curve), 100 (green curve), and 10,500 (black curve) OL iterations.

explained by the lack of convergence of the nonlinear approximate solutions.

[46] These results point out to the fact that the slow convergence is related to the internal ice stress term. Indeed, the large velocity errors are found in regions where the ice-ice and ice-continent interactions dominate and are not observed in regions where the ice interactions are less important (e.g., close to the ice edge). The water drag, the other nonlinear term in the momentum equation is not responsible for the slow convergence of the nonlinear solution. When treating the water drag as a linear term $\left(\left|\mathbf{u}-\mathbf{u}_{w}^{g}\right|\right.$ is kept constant at $10 \mathrm{~cm} \mathrm{~s}^{-1}$ in equation (12)), the slow convergence is still present. On the other hand, when setting the viscous coefficients to a constant value $\left(\zeta=1 \times 10^{12} \mathrm{kgs}^{-1}\right)$ and keeping the water drag nonlinear, the FC solution is obtained in only 30 OL iterations.

\section{Considerations on the VP Formulation and the Nonlinear Solver}

\subsection{Numerical Convergence of the Approximate Solution}

[47] A theoretical explanation for the observed slow convergence is beyond the scope of this paper. The sea ice momentum equation is intrinsically a difficult equation to solve owing to its high nonlinearity. We argue however that the convergence properties can be improved by (1) making the momentum equation continuously differentiable and (2) using the Newton method for the nonlinear solver. Note that for this former point, the rheology term in the momentum equation is not continuously differentiable owing to the capping of the viscous coefficients (see equations (8) and (9)).

[48] First, we replace the expression of $\zeta$ (equation (8)) by a smooth formulation written as

$$
\zeta=\zeta_{\max } \tanh \left(\frac{P}{2} \triangle^{-1} \zeta_{\max }^{-1}\right)+\zeta_{\min }
$$

[49] As in equation (7), $\eta=\zeta e^{-2}$. The two formulations of the bulk viscosity are shown on Figure 10a on a log-log plot for $h=1 \mathrm{~m}$ and $A=1$. The model was run with the new formulation for the same time step previously studied (6 January 19970000 UT). The solid curve on Figure 10b shows the residual norm of the nonlinear system of equations as a function of the number of OL iterations when the "standard" formulation of $\zeta$ and $\eta$ is used (the same curve as in Figure 1) and the dashed curve when the "tanh" formulation of $\zeta$ and $\eta$ is used (equation (14)). Note that the FC velocity fields and deformations fields are very similar (results not shown) with the two approaches. Other formulations used in the community also result in a continuously differentiable rheology term [e.g., Kreyscher et al., 2000].

[50] Figure $10 \mathrm{~b}$ shows that $\sim 4500 \mathrm{OL}$ iterations are necessary to obtained the FC solution when the rheology term is continuously differentiable as opposed to $\sim 10,500$ when the standard formulation is used. This result is robust. The number of $\mathrm{OL}$ iterations required to reach the $\mathrm{FC}$ solution is always significantly less when using the tanh formulation as opposed to the standard formulation. The same conclusion applies when using the $110-\mathrm{km}$ resolution model.

[51] Second, to highlight the difference between the Newton method and the nonlinear solver used in VP models (referred to as the standard approach), consider the simple one dimensional nonlinear equation:

$$
F(u)=\zeta(u) u-b=0
$$

[52] In this equation, the term $\zeta(u) u$ mimics the rheology term. With a Taylor expansion and neglecting higher-order terms, we write

$$
F(u+\delta u) \approx F(u)+F^{\prime}(u) \delta u
$$


a

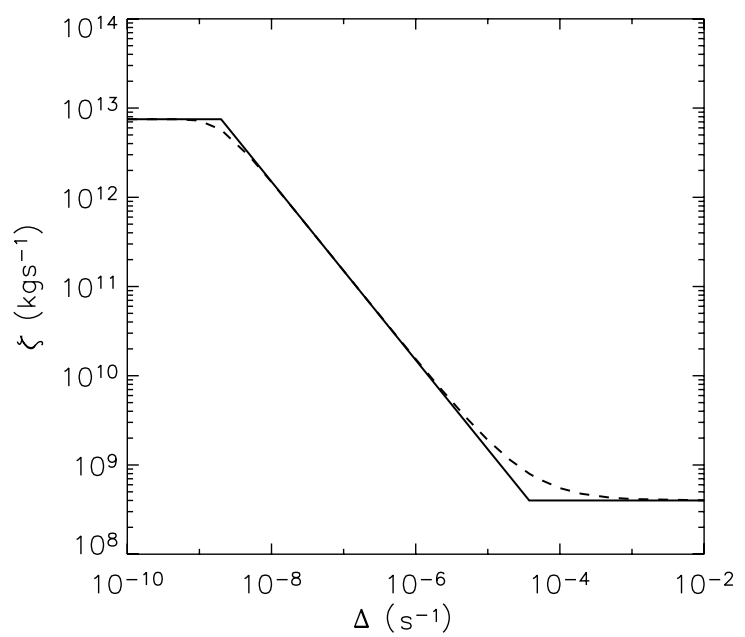

b

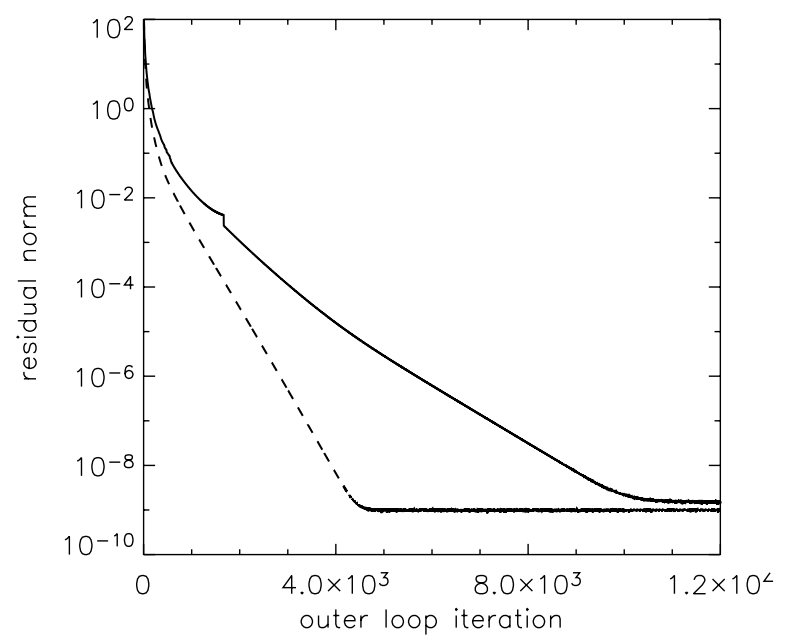

Figure 10. (a) Two formulations of the bulk viscosity $\zeta$ as a function of the deformation $\Delta$ (see equation (6)). The solid curve is the standard formulation of Hibler [1979] and the dashed curve is the formulation with the tanh (equation (14)). (b) Residual norm of the nonlinear system of equations on 6 January 19970000 UT as a function of the OL iteration when the standard (solid curve) and the tanh (dashed curve) formulation of $\zeta$ and $\eta$ is used. The residual norms are calculated over the whole domain.

where the prime denotes the derivative with respect to $u$. In the Newton approach, the correction $\delta u$ is obtained by requiring that $F(u+\delta u)=0$. At iteration $k$, we then write

$$
\delta u^{k}=u^{k}-u^{k-1}=\frac{-F\left(u^{k-1}\right)}{F^{\prime}\left(u^{k-1}\right)} .
$$

Differentiating equation (15), with respect to $u$ and substituting into equation (17), we obtain:

$$
u^{k}=u^{k-1}-\frac{F\left(u^{k-1}\right)}{\zeta^{\prime}\left(u^{k-1}\right) u^{k-1}+\zeta\left(u^{k-1}\right)} .
$$

In the standard approach (used in all current VP models with an implicit solver), the linearization process considers that $\zeta^{\prime}(u)=0$. The kth iterate of the velocity is therefore

$$
u^{k}=u^{k-1}-\frac{F\left(u^{k-1}\right)}{\zeta\left(u^{k-1}\right)} .
$$

Note that for comparison with the Newton method, $u^{k-1}$ is used for the linearization as opposed to $\left(u^{k-1}+u^{k-2}\right) / 2$ (equation (13)).

[53] Both approaches (equations (18) and (19)) are fixed point iterations as they can be written as $u^{k}=\phi\left(u^{k-1}\right)$ [Quarteroni et al., 2000]. However, we argue that the common practice of assuming that $\zeta^{\prime}(u)=0$ partly explains the slow convergence rate. Theoretically, with the Newton method, $u^{k}$ converges quadratically to the solution $u$ if $u^{k}$ is sufficiently close to $u$ and if $F^{\prime}$ is Lipschitz continuous at $u$ [Eisenstat and Walker, 1994]. This is however one drawback of the Newton method: the convergence is quadratic in the vicinity of the solution (local convergence) but obtaining the solution from any initial guess (global convergence) can be difficult for some problems.

\subsection{Uniqueness of the Solution}

[54] Hunke [2001] has theoretically demonstrated, for an idealized case (no forcing), that multiple solutions can exist with the VP formulation. In a realistic experiment, we have observed the existence of multiple solutions. When low values of $\alpha(k)$ (undersolving) are used in the tolerance evolution, the approximate solution converges to a FC solution but this FC solution is not the same as the one obtained with the optimal progressive $\alpha(k)$ tolerance. Figure 11a shows the residual norm of the nonlinear system of equations on 6 January 19970000 UT as a function of the OL iteration when the optimal progressive $\alpha(k)$ tolerance (solid curve) is used and when a constant $\alpha$ of 10 is used (dashed curve). In both cases, the approximate solution is limited by the machine precision. Figure $11 \mathrm{~b}$ shows the velocity difference between the FC velocity field obtained with $\alpha=10$ and the one obtained with the optimal progressive $\alpha(k)$ tolerance.

[55] There are differences of $\mathrm{O}\left(10^{-2} \mathrm{~cm} \mathrm{~s}^{-1}\right)$ in some parts of the domain. When $\alpha>25$, the approximate solution always converges to the FC solution obtained with the optimal progressive $\alpha(k)$ tolerance. Note that this issue of multiple solutions is not present when using the tanh formulation (which leads to a continuously differentiable momentum equation).

\section{Conclusion}

[56] We have found that the numerical convergence of the nonlinear solver used in VP models is slow. When a small number of OL iterations are performed when using a $6 \mathrm{~h}$ time step, the errors associated with the velocity field approximate solution are of the same order of magnitude as the mean drift. Moreover, we have found that the metrics (average kinetic energy of the pack and VP solution) used in the sea ice modeling community to assess convergence of the approximate solution are misleading. 
a

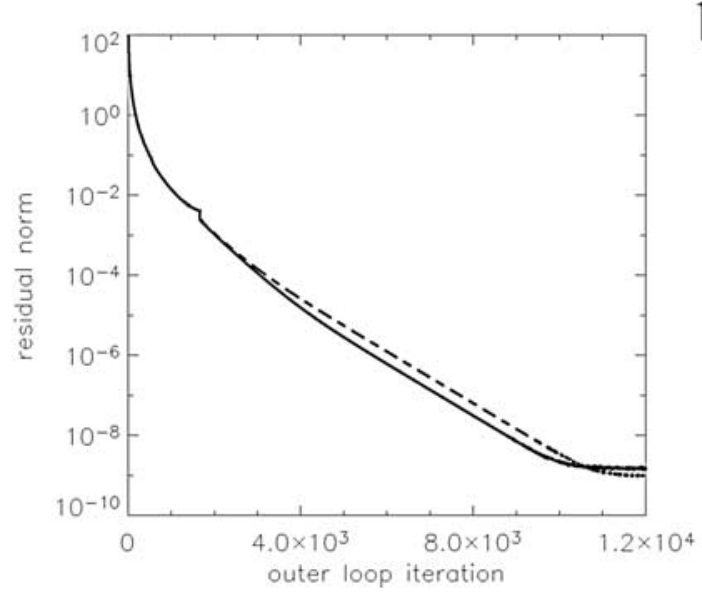

$\mathrm{b}$

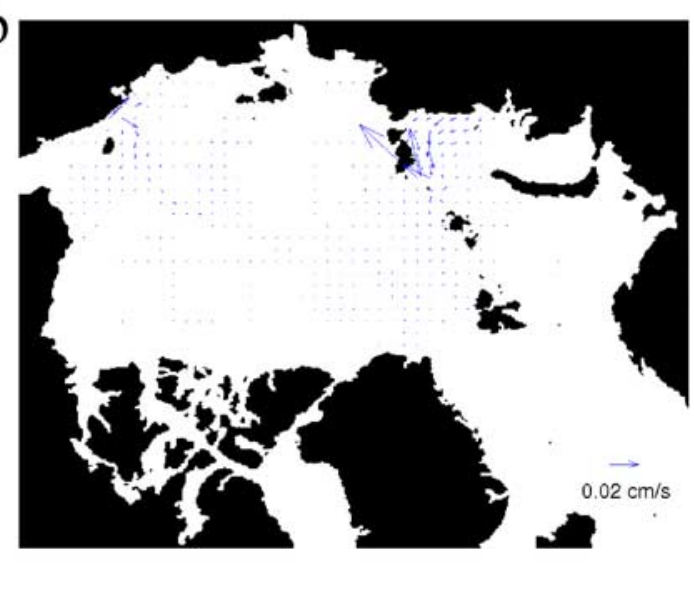

Figure 11. (a) Residual norm of the nonlinear system of equations on 6 January 19970000 UT as a function of the OL iteration when the optimal progressive $\alpha(k)$ tolerance (solid curve) is used and when a constant $\alpha$ of 10 is used (dashed curve). The residual norms are calculated over the whole domain. (b) Difference between the FC velocity field obtained with $\alpha=10$ and the FC velocity field obtained with the optimal progressive $\alpha(k)$ tolerance.

[57] When performing two OL iterations (equivalent to a single modified Euler time step) with a $10-\mathrm{km}$ resolution model with a $6 \mathrm{~h}$ time step and forced by 6 hourly varying wind stress, the simulated velocity field exhibits errors as large as $15 \mathrm{~cm} \mathrm{~s}^{-1}$ and errors of $\sim 5 \mathrm{~cm} \mathrm{~s}^{-1}$ are present in large regions of the domain. For a small number of OL iterations, the majority of the states of stress are inside the yield curve (viscous) and a significant amount of the stresses are unrealistic (outside the yield curve).

[58] It was previously shown that when performing $10 \mathrm{OL}$ iterations, the average kinetic energy of the pack is always within $2 \%$ of the fully converged (FC) value [Lemieux et al., 2008]. When using this criterion, we have found that the errors on the drift are of the same order of magnitude as the mean drift. Also, the states of stress in that case do not exhibit a VP solution.

[59] As more and more OL iterations are performed, the states of stress migrate from viscous states to plastic states and the unrealistic states of stress move from outside of the yield curve to the inside of the yield curve and its contour. While 40 OL iterations does provide a VP solution, there are still errors on the drift larger than $1 \mathrm{~cm} \mathrm{~s}^{-1}$ at some locations on the domain and large parts of the central Arctic are characterized by errors of $\sim 0.5 \mathrm{~cm} \mathrm{~s}^{-1}$.

[60] With a small time step (30 $\mathrm{min})$ compared to the forcing time scale and using the previous time step solution as the initial guess, the quality of the approximate solution is greatly improved when compared to the $6 \mathrm{~h}$ time step case (for the same number of OL iterations). A significant improvement is also seen in the simulated monthly mean fields when a 30 min time step is used.

[61] The errors in the velocity field are not random: they do not average out with time. When performing two OL iterations, the monthly mean velocity field (January 1997) has errors of the same order of magnitude as the mean drift itself. This lack of convergence of the approximate velocity field solution causes sea ice buildup in some regions and thinner ice in others.

[62] After $40 \mathrm{OL}$ iterations, there are velocity errors of $0.5-1 \mathrm{~cm} \mathrm{~s}^{-1}$ concentrated along shear lines. They are associated with large sea ice deformations occurring in regions where ice interactions dominate. Subsequent OL iterations cause the states of stress to slowly migrate while remaining inside and on the yield curve. During this process, the shear lines become better defined (with higher maximum value of shear strain rate at the shear line and lower shear between shear lines) and sometimes relocalize spatially in a nearby location. This is also observed in the divergence field.

[63] In a recent paper, Kwok et al. [2008] found that current sea ice models systematically underestimate deformations (particularly divergence) when compared to Radarsat derived deformations. We raise the question wether the discrepancy between data and simulated deformations could be partly explained by the lack of convergence of the nonlinear approximate solutions.

[64] These conclusions are robust. The slow convergence of the approximate solution is an issue at all spatial resolutions but is more severe as the grid is refined. It also does not depend on the linear solver used. It is the rheology term, not the water drag term, in the momentum equation that is responsible for the slow convergence. The convergence rate of the approximate solution can be improved by (1) making the momentum equation continuously differentiable and (2) using the Newton method for the nonlinear solver. It was also observed that this discontinuity in the momentum equation can also lead to the existence of multiple solutions.

[65] The effect of these errors on climate simulations and a criterion for an acceptable level of errors remain to be investigated. Nevertheless, it appears that the development of a more computationally efficient nonlinear solver that would allow one to obtain a sufficiently converged approximate solution in a smaller number of iterations is desirable.

[66] We are currently developing a globally convergent Newton method to improve the convergence rate. In a problem of $n$ dimensions, $F^{\prime}$ is the Jacobian $(\mathbf{J})$, an $n \times n$ matrix. At each Newton iteration, the system of equations is written as $\mathbf{J}\left(\mathbf{u}^{\mathbf{k}-\mathbf{1}}\right) \delta \mathbf{u}^{\mathbf{k}}=-\mathbf{F}\left(\mathbf{u}^{\mathbf{k}-\mathbf{1}}\right)$ which we want to solve for $\delta \mathbf{u}^{\mathbf{k}}$. This is a matrix-free method; that is, the Jacobian matrix never needs to be formed explicitly. Only the product 
of $\mathbf{J}$ times a vector is needed. However, in our problem, $\mathbf{J}$ times a vector is difficult to obtain because of the complexity of the momentum equation and mainly because of the complicated land-ocean configuration (this is especially difficult when using a $\mathrm{C}$ grid). We are developing instead a Jacobian free Newton-Krylov method [Knoll and Keyes, 2004]. This will involve a Newton iteration (in which $\mathbf{J}$ is approximated) along with the already developed preconditioned GMRES method. To ensure global convergence, a line search method (a backtracking method) is used [Eisenstat and Walker, 1994]. Preliminary results show that compared to the standard solver, the JFNK method reduces by a factor of 10 the number of iterations required to reach a certain residual norm.

[67] Acknowledgments. We would like to thank Martin Losch, Einar Olason, Jan Sedláček, Stephen Thomas, and Paul Tupper for many useful discussions during the course of this work. We also want to thank Robert Pritchard and an anonymous reviewer for useful comments. Jean-François Lemieux would like to thank NSERC, FQRNT, and the McGill University J.W. McConnell foundation for fellowships received during the course of this project. Bruno Tremblay is grateful to NSERC for a Discovery grant, and to the National Science Foundation Office of Polar Program (OPP0230325) and Arctic Science Program (ARC-0520496). This work was also partially supported by an NSERC Discovery grant and NSERC/CFCASfunded Canadian CLIVAR Research Network grant awarded to Lawrence Mysak.

\section{References}

Arbetter, T. E., J. A. Curry, and J. A. Maslanik (1999), Effects of rheology and ice thickness distribution in a dynamic-thermodynamic sea ice model, J. Phys. Oceanogr., 10, 2656-2670.

Dukowicz, J. (1997), Comments on the "Stability of the viscous-plastic sea ice rheology", J. Phys. Oceanogr., 27, 480-481.

Eisenstat, S. C., and H. F. Walker (1994), Globally convergent inexact Newton methods, SIAM J. Optim., 4, 393-422.

Geiger, C. A., W. D. Hibler, and S. F. Ackley (1998), Large-scale sea ice drift and deformation: Comparison between models and observations in the western Weddell Sea during 1992, J. Geophys. Res., 103, 21,89321,913 .

Hibler, W. D. (1979), A dynamic thermodynamic sea ice model, J. Phys. Oceanogr., 9, 815-846.

Hibler, W. D., and S. F. Ackley (1983), Numerical simulation of the Weddell Sea pack ice, J. Geophys. Res., 88, 2873-2887.

Hibler, W. D., and J. E. Walsh (1982), On modeling seasonal and interannual fluctuations of Arctic sea ice, J. Phys. Oceanogr., 12, 1514-1523.

Hunke, E. C. (2001), Viscous-plastic sea ice dynamics with the EVP model: Linearization issues, $J$. Comput. Phys, $170,18-38$.

Hunke, E. C., and J. K. Dukowicz (1997), An elastic-viscous-plastic model for sea ice dynamics, J. Phys. Oceanogr., 27, 1849-1867.

Hunke, E. C., and J. K. Dukowicz (2002), The elastic-viscous-plastic sea ice dynamics model in general orthogonal curvilinear coordinates on a sphere: Incorporation of metric terms, Mon. Weather Rev., 130, 1848 1865.
Hunke, E. C., and Y. Zhang (1999), A comparison of sea ice dynamics models at high resolution, Mon. Weather Rev., 127, 396-408.

Hutchings, J. K., P. Heil, and W. D. Hibler (2005), Modeling linear kinematic features in sea ice, Mon. Weather Rev., 133, 3481-3497.

Ip, C. F. (1993), Numerical investigation of different rheologies on sea-ice dynamics, Ph.D. thesis, 242 pp., Darmouth Coll., Hanover, N. H.

Ip, C. F., W. D. Hibler, and G. M. Flato (1991), On the effect of rheology on seasonal sea-ice simulations, Ann. Glaciol., 15, 17-25.

Kalnay, E., et al. (1996), The NCEP/NCAR 40-year reanalysis project, Bull. Am. Meteorol. Soc., 77, 437-470.

Knoll, D. A., and D. E. Keyes (2004), Jacobian-free Newton-Krylov methods: A survey of approaches and applications, J. Comput. Phys., 193, 357-397.

Kreyscher, M., M. Harder, P. Lemke, and G. M. Flato (2000), Results of the Sea Ice Intercomparison Project: Evaluation of sea ice rheology schemes for use in climate simulations, J. Geophys. Res., 105, 11,299-11,320.

Kwok, R. (2001), Deformations of the Arctic Ocean sea ice cover between November 1996 and April 1997: A survey, in IUTAM Symposium on Scaling Laws in Ice Mechanics and Ice Dynamics: Proceedings of the IUTAM Symposium Held in Fairbanks, Alaska, USA, 13-16 June 2000 , edited by J. Dempsey and H. Shen, pp. 301-322, Kluwer Acad., Dordrecht.

Kwok, R., E. C. Hunke, D. Maslowski, and J. Zhang (2008), Variability of sea ice simulations assessed with RGPS kinematics, J. Geophys. Res. 113, C11012, doi:10.1029/2008JC004783.

Lemieux, J.-F., B. Tremblay, S. Thomas, J. Sedláček, and L. A. Mysak (2008), Using the preconditioned Generalized Minimum RESidual (GMRES) method to solve the sea-ice momentum equation, J. Geophys. Res., 113, C10004, doi:10.1029/2007JC004680.

Maslowski, W., and W. H. Lipscomb (2003), High resolution simulations of Arctic sea ice, 1979-1993, Polar Res., 22, 67-74.

McPhee, M. G. (1975), Ice-ocean momentum transfer for the AIDJEX ice model, AIDJEX Bull., 29, 93-111.

McPhee, M. G., R. Kwok, R. Robbins, and M. Coon (2005), Upwelling of Arctic pycnocline associated with shear motion of sea ice, Geophys. Res. Lett., 32, L10616, doi:10.1029/2004GL021819.

Oberhuber, J. (1993), Simulation of the Atlantic circulation with a coupled sea ice-mixed layer-isopycnal general circulation model. Part I: Model description, J. Phys. Oceanogr., 23, 808-829.

Quarteroni, A., R. Sacco, and F. Saleri (2000), Numerical Mathematics, Springer, New York.

Riedlinger, S. H., and R. H. Preller (1991), The development of a coupled ice-ocean model for forecasting ice conditions in the Arctic, J. Geophys. Res., 96, 16,955-16,977.

Schmidt, G., et al. (2006), Present-day atmospheric simulations using GISS ModelE: Comparison to in situ, and reanalysis data, J. Clim., 19, $153-$ 192.

Tremblay, L.-B., and M. Hakakian (2006), Estimating the sea ice compressive strength from satellite-derived sea ice drift and NCEP reanalysis data, J. Phys. Oceanogr., 36, 2165-2172.

Tremblay, L.-B., and L. A. Mysak (1997), Modeling sea ice as a granular material, including the dilatancy effect, J. Phys. Oceanogr., 27, $2342-$ 2360

Zhang, J., and W. Hibler (1997), On an efficient numerical method for modeling sea ice dynamics, J. Geophys. Res., 102, 8691-8702.

Zhang, J., and D. A. Rothrock (2000), Modeling Arctic sea ice with an efficient plastic solution, J. Geophys. Res., 105, 3325-3338.

J.-F. Lemieux and B. Tremblay, Department of Atmospheric and Oceanic Sciences, McGill University, 805 Sherbrooke Street West, Montreal, QC H3A 2K6, Canada. (lemieux@zephyr.meteo.mcgill.ca) 